C-A/AP/\#146

March 2004

\title{
Observations on background in PHOBOS and related electron cloud simulations
}

\author{
G. Rumolo, GSI \\ W. Fischer, BNL
}

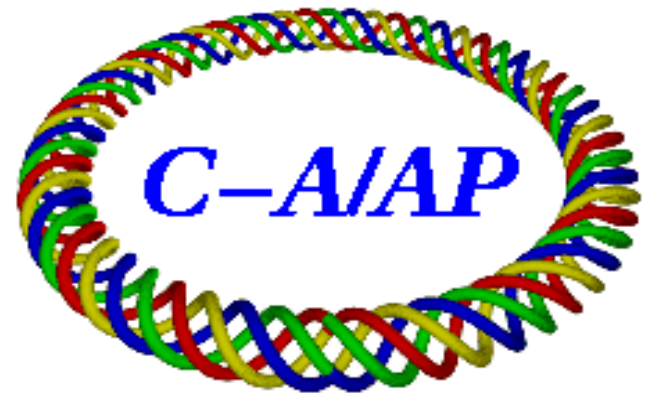

Collider-Accelerator Department Brookhaven National Laboratory Upton, NY 11973 


\title{
Observations on background in PHOBOS and related electron cloud simulations
}

\author{
G. Rumolo \\ GSI-Darmstadt, Planckstrasse 1, 64291 Darmstadt (Germany) \\ W. Fischer \\ BNL, Upton, New York 11973 (USA)
}

\section{Abstract}

In this report we summarize the observations of pressure rise and background problems in PHOBOS, and we investigate the possibility of electron multipacting as a plausible explanation of these phenomena. PHOBOS has a beryllium pipe that extends $6 \mathrm{~m}$ to either side of the Interaction Point (IP), and is therefore likely to suffer from electron cloud even when RHIC is operating with rather large bunch spacing, due to the very high maximum Secondary Emission Yield (SEY) of beryllium [Hilleret00]. A simulation study of electron cloud in the beryllium pipe of PHOBOS covering wide ranges of parameters is carried out using the ECLOUD code [Rumolo02].

\section{PHOBOS background in RHIC Run-4}

Trains of 45, 56 or 61 bunches have been injected and ramped in RHIC during Run-4. At each ramp a first pressure rise is observed at transition crossing, likely to be caused by the bunch shortening (bunches are about 4 ns long when going through transition, between the $20 \mathrm{~ns}$ at injection and the $10 \mathrm{~ns}$ at top energy before rebucketing). Shortly after transition, the pressure is restored to its original value, as the bunches get longer again. At storage energy, the bunches are transferred from the accelerating rf system, with 36 ns bucket length, to the storage rf system, with $5 \mathrm{~ns}$ bucket length. The bunch rotation process used for rebucketing halves the bunch length and doubles the peak intensity. A second pressure rise may occur after rebucketing and can stay for about one hour. Some peculiar features of this effect point to electron clouds as the most probable cause of the pressure rise:

- The bunch length dependence. The pressure rises when the bunch length is halved and the bunch peak intensity is doubled.

- The persistence over an extended time. After some random time (spanning between a few minutes and over one hour) the pressure subsides indicating that some threshold has been crossed again. We can suppose that at this point the multipacting is switched off and the electron cloud disappears. The beam currents at which the pressure drops vary by some $25 \%$ around a value that is about $90 \%$ of the current at rebucketing. 
- The surface properties in PHOBOS. In this region, a $12 \mathrm{~m}$ beryllium pipe with a $3.6 \mathrm{~cm}$ radius is placed. Beryllium is known to have a very high maximum SEY ( $\delta_{\max }=2.8$ [Hilleret00]), which can easily cause electron accumulation even for relatively large bunch spacings and moderate bunch intensities.

- The independence of the beam energy. The PHOBOS pressure rise occurs at both $100.0 \mathrm{GeV} / \mathrm{u}$ and $31.2 \mathrm{GeV} / \mathrm{u}$ beam energy in gold-gold operation. An electron cloud should only depend on the charge, not the beam energy.

The pressure rise causes an intolerable background in PHOBOS.

Fig. 1 shows a typical filling with 56 bunches exhibiting the pressure rise in PHOBOS after rebucketing. The filling pattern with 56 bunches is regular and bunches are separated by 6 buckets with a 24 bucket abort gap at the end of the train. With 56 or more bunches the machine can exceed and normally runs close to the vacuum limits in Blue sector 8 (unbaked collimator), and Yellow sector 4 (stochastic cooling kicker, baked only during the run).

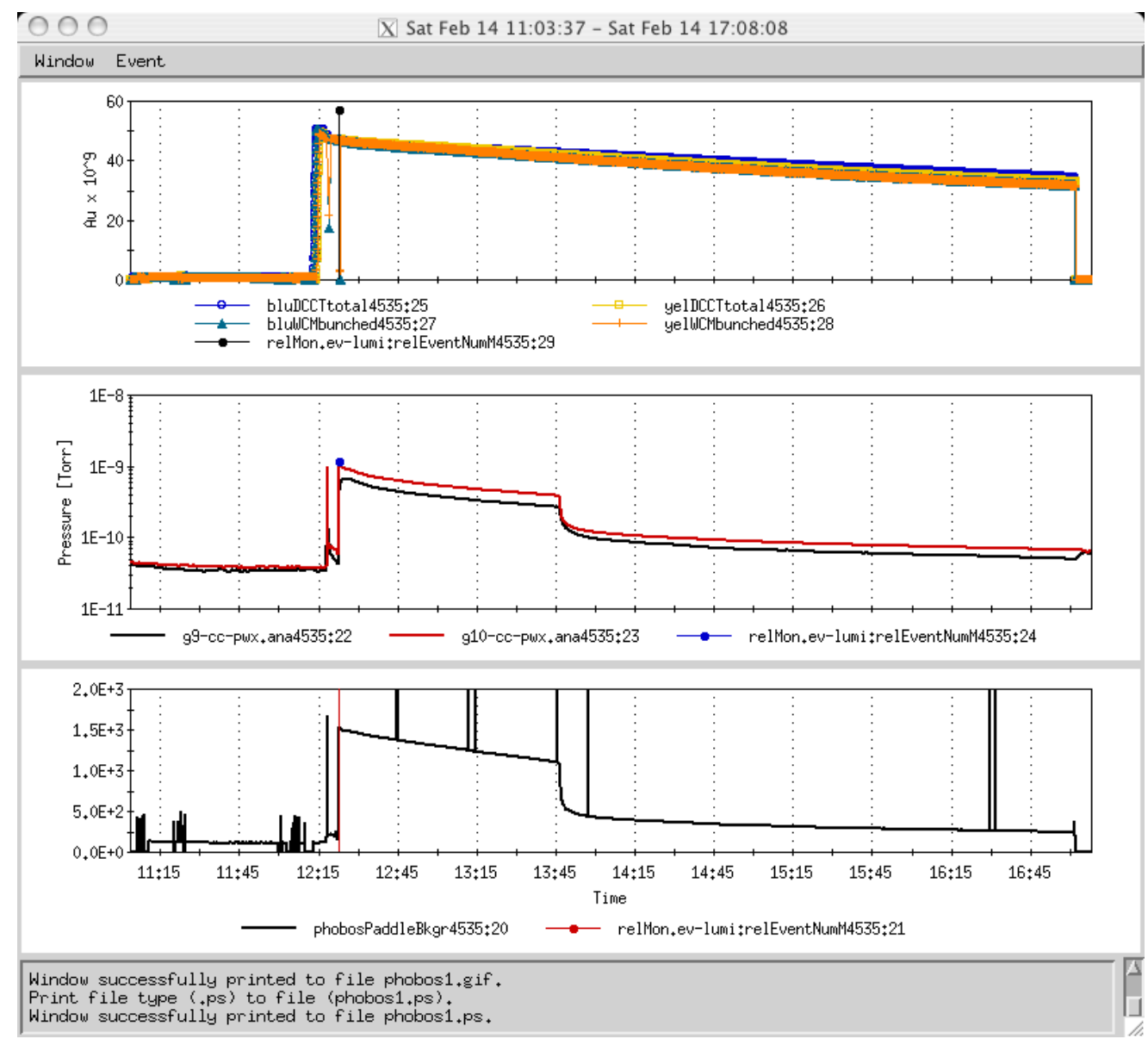

Fig. 1 Pressure rise in PHOBOS after rebucketing with a 56 bunch filling. The curves show respectively: the current evolution in the Yellow and Blue rings with the rebucketing event marked with a vertical line (upper), the pressure evolution read from two gauges situated in IR10 left and right of the IP (middle) and the background signal (lower). 
When RHIC is operated with 61 bunches, most bunches are separated by 6 buckets and some by 3 buckets. When RHIC is operated with 45, most bunches are separated by 6 buckets and some by 9 buckets. The bunches are still distributed as uniformly as possible to minimize electron cloud effects, and have a 3-fold symmetry to provide approximately an equal amount of bunchbunch collisions for all of the four experiments [Fischer03]. For 45 bunches per ring more intensity per bunch is required in order to maintain the luminosity as with 56 bunches. This puts more demand on the injector chain. With 45 bunches, the luminosity is limited by the bunch intensity available from the injectors. Thus, whenever the injectors cannot provide enough bunch intensity, the bunch number is increased.

In order to maximize the luminosity, the bunch number was increased from 56 to 61 early in the run. After about one week of running with 61 bunches, pressure rises were observed at PHOBOS, and the bunch number was reduced again to 56 and the pressure rises were suppressed. Two weeks later the problem resurfaced. The bunch number was then reduced to 45 bunches. This initially reduced the number of stores with a pressure problem, but the situation gradually deteriorated again, and after another 4 weeks almost all stores showed a pressure rise at PHOBOS. Figure 2 shows typical 45 bunch fillings with and without the background problem. Fig. 3 summarizes the history of the PHOBOS pressure rise problem in Run-4.
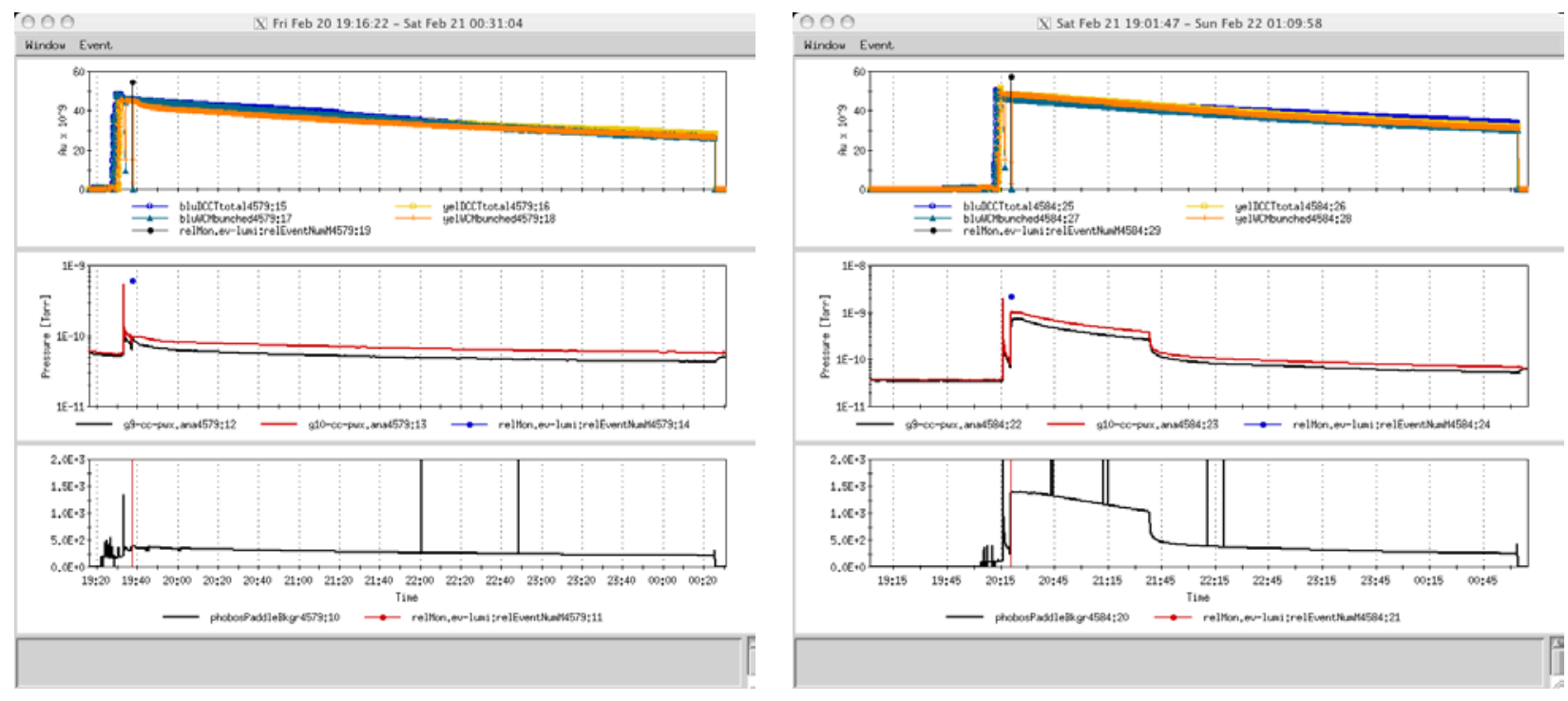

Fig. 2 Pressure rise in PHOBOS after rebucketing for a 45 bunch filling without (left) and with background problem (right). The curves show respectively: the current evolution in the Yellow and Blue rings with the rebucketing event marked with a vertical line (upper), the pressure evolution read from two gauges situated in the IR10 left and right of the IP (middle) and the background signal (lower).

The static pressure in Phobos has more than doubled (from 20 up to a maximum of 80 pTorr) since the background problem appeared. This may be either the cause or the result of the pressure rise. A leak check with Argon at Phobos has not revealed any leaks in the beam pipe areas that were accessible. A more thorough leak check with Helium is planned for the shutdown period between the $\mathrm{Au}-\mathrm{Au}$ and $\mathrm{p}-\mathrm{p}$ runs. 

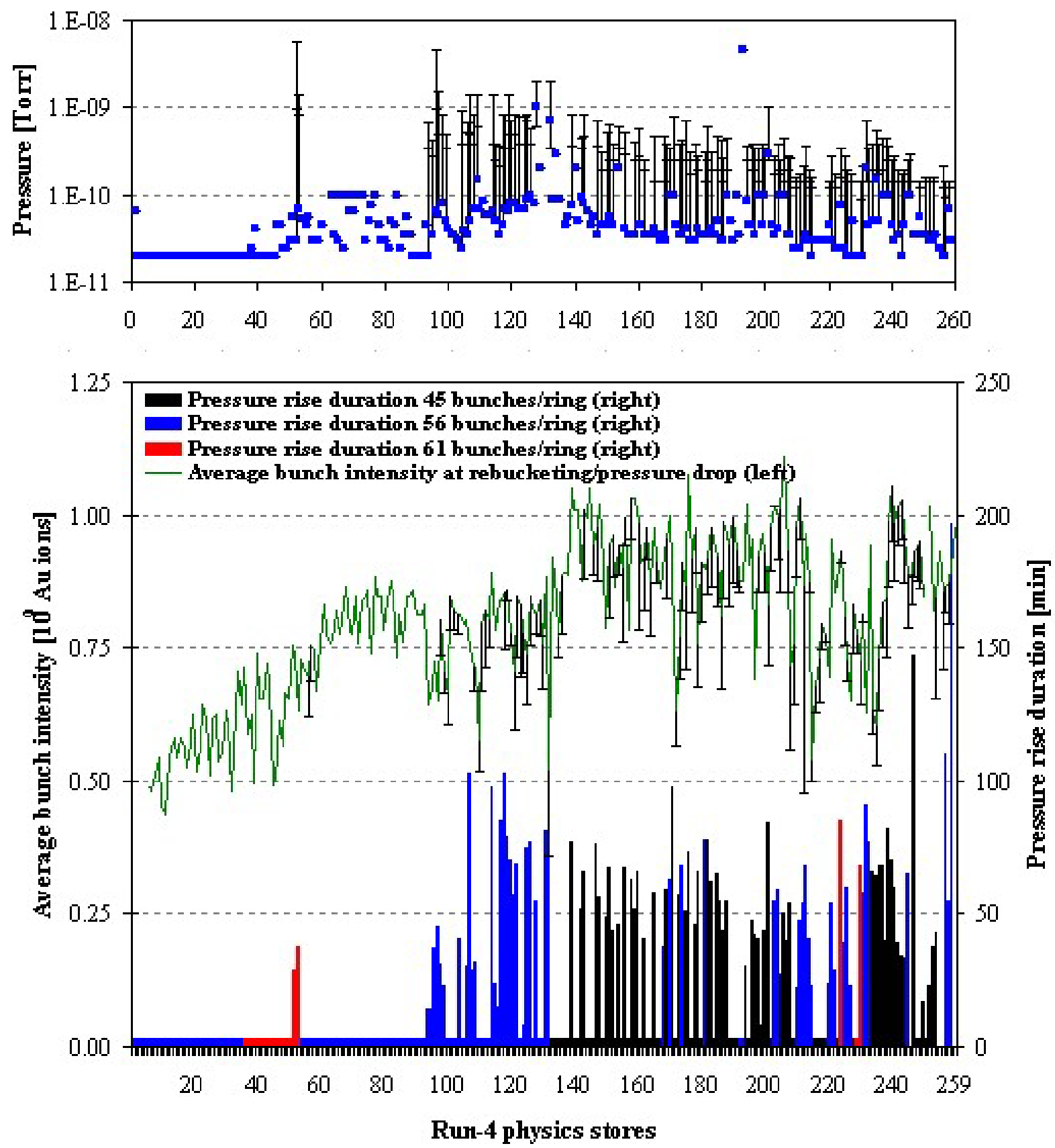

Fig. 3 History of the PHOBOS pressure rise during the RHIC Run-4. In the upper part, the pressure in IR10 is shown at rebucketing for the physics stores of Run-4. For stores with a pressure rise after rebucketing, also shown are the maximum pressure and the pressure when it begins to drop sharply. In the lower part on the left scale the bunch intensity, averaged over all bunches in the Blue and Yellow rings, is depicted. Stores with a PHOBOS vacuum problem also show the average bunch intensity at the time when the pressure begins to drop sharply. In the lower part on the right scale, the duration of the pressure problem is shown, ordered into stores with 45,56 , and 61 bunches per ring. Note that the last 14 stores are with beams at $31.2 \mathrm{GeV} / \mathrm{u}$, all other stores are with beams of $100.0 \mathrm{GeV} / \mathrm{u}$. 


\section{Electron cloud build up simulations}

In order to check on the possibility that the pressure rise in PHOBOS is caused by an electron cloud inside the beryllium pipe, we have carried out a number of simulations with the ECLOUD code [Rumolo02], sweeping large ranges of parameters. The standard parameters that we used of in our simulations are summarized in the Table below.

\begin{tabular}{|l|c|c|c|}
\hline & Symbol and unit & Before rebucketing & After rebucketing \\
\hline Ring circumference & $C(\mathrm{~m})$ & 3833 \\
\hline Ions/bunch $\left(\mathrm{Au}^{79+}\right)$ & $N_{b}$ & 2 & 1 \\
\hline Rms bunch length & $\sigma_{z}(\mathrm{~ns})$ & 100 \\
\hline Energy & $E(\mathrm{GeV} / \mathrm{u})$ & 2.5 \\
\hline Transverse norm. rms emittance & $\varepsilon_{x, y}(\mu \mathrm{m})$ & 3 \\
\hline Beta function at the IP & $\beta^{*}(\mathrm{~m})$ & 11 \\
\hline Beta at the end of the Be & $\beta(\mathrm{s})(\mathrm{m})$ & $40-100$ \\
\hline Static pressure & $P(\mathrm{pTorr})$ & 3.61 \\
\hline Radius of the Be chamber & $r_{c}(\mathrm{~cm})$ & 2.8 \\
\hline Maximum SEY of Be & $\delta_{\max }$ & 250 \\
\hline Energy of the maximum SEY & $E_{\max }(\mathrm{eV})$ & 2 \\
\hline
\end{tabular}

As we are in the neighborhood of the IP, bunches that go through each section can have in principle any arbitrary separation spanning between the half spacing with nominal intensity and the full spacing with double intensity (exactly at the IP). In our simulation campaign we have started with considering the two extreme cases: nominal spacing with doubled intensity (bunches meeting at the IP) and non-uniform spacing with nominal intensity (at the end of the beryllium pipe). In the latter case bunches are spaced by $12 \mathrm{~m}$ and $52 \mathrm{~m}$ in the 56 bunch operation, and have a non-uniform $(12,52) \times 4+(12,84) \times 2$ bunch separation in the 45 bunch operation. Bunch shapes at the IP and at the end of the beryllium pipe are shown in Fig. 4. Subsequently, we have refined our analysis and considered various mixed filling patterns in order to simulate the electron cloud build up at different locations inside the beryllium pipe. We list what we have considered in our simulations (assumptions and scans):

- A field-free region

- A beryllium pipe with its surface parameters, subsequently adjusted to match the observations.

- Filling patterns with 56 and 45 bunches.

- Longitudinal positions at the IP, and a few selected locations along the beryllium pipe, including the end.

- Off-centered beams.

- Different currents to investigate threshold behavior.

Below we list what we have neglected in our analysis (possibly second order effects): 
- The abort gaps do not meet at the PHOBOS location, so there is actually always some beam going through the IR.

- After rebucketing bunches can be longitudinally mismatched and execute quadrupole synchrotron oscillations. We believe that neglecting this effect should not produce any significant error, because the electron cloud builds up within a few turns, whereas the synchrotron period is hundreds of turns.

- Quadrupole field at the IP.
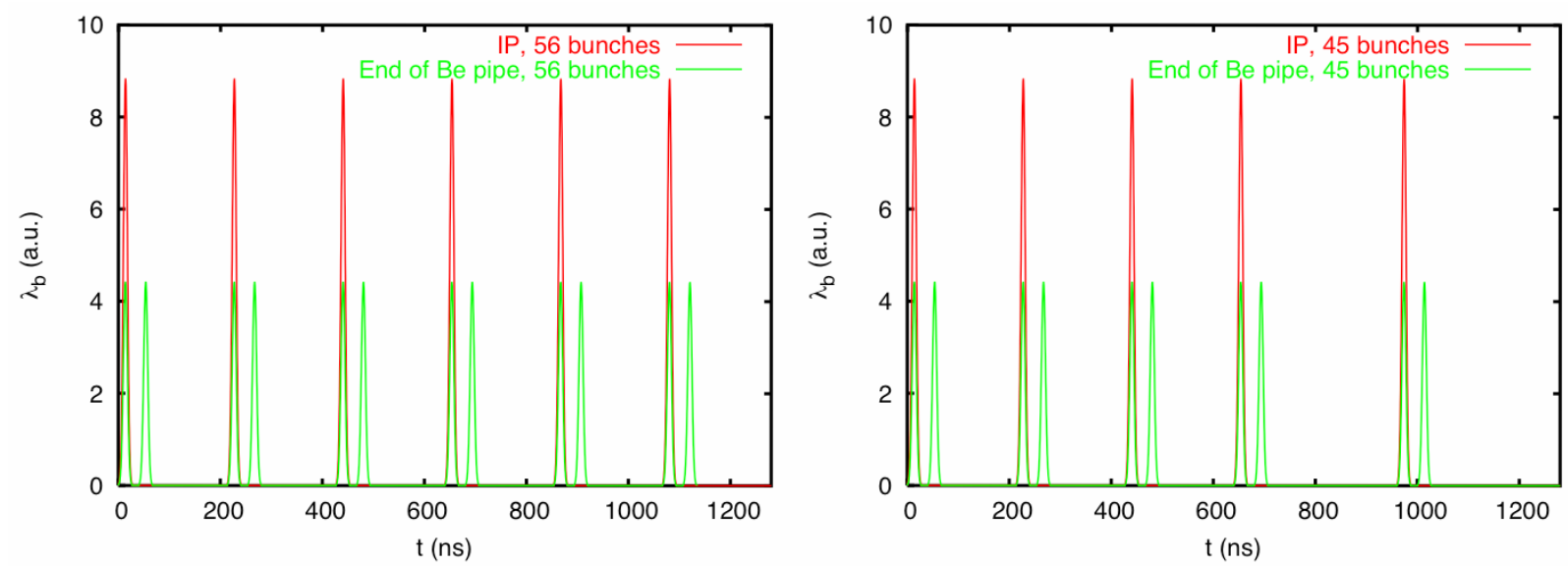

Fig. 4 Left: bunches in the 56-bunch configuration at the IP and at the end of the Be pipe (abort gap is 24 buckets). Right: bunches in the 45-bunch configuration at the IP and at the end of the Be pipe (abort gap is 30 buckets).

Simulations show that if we simply consider $\delta_{\max }$ to be 2.8 and $\mathrm{E}_{\max }=250 \mathrm{eV}$ (Fig. 5, red curve), we would expect an electron cloud in the everywhere in the beryllium pipe for both before and after rebucketing, and for both 45 and 56 bunches per ring. This is illustrated in Fig. 6 (left side). However, we can adjust $\delta_{\max }$ and $\mathrm{E}_{\max }$ to match some of the PHOBOS observations.

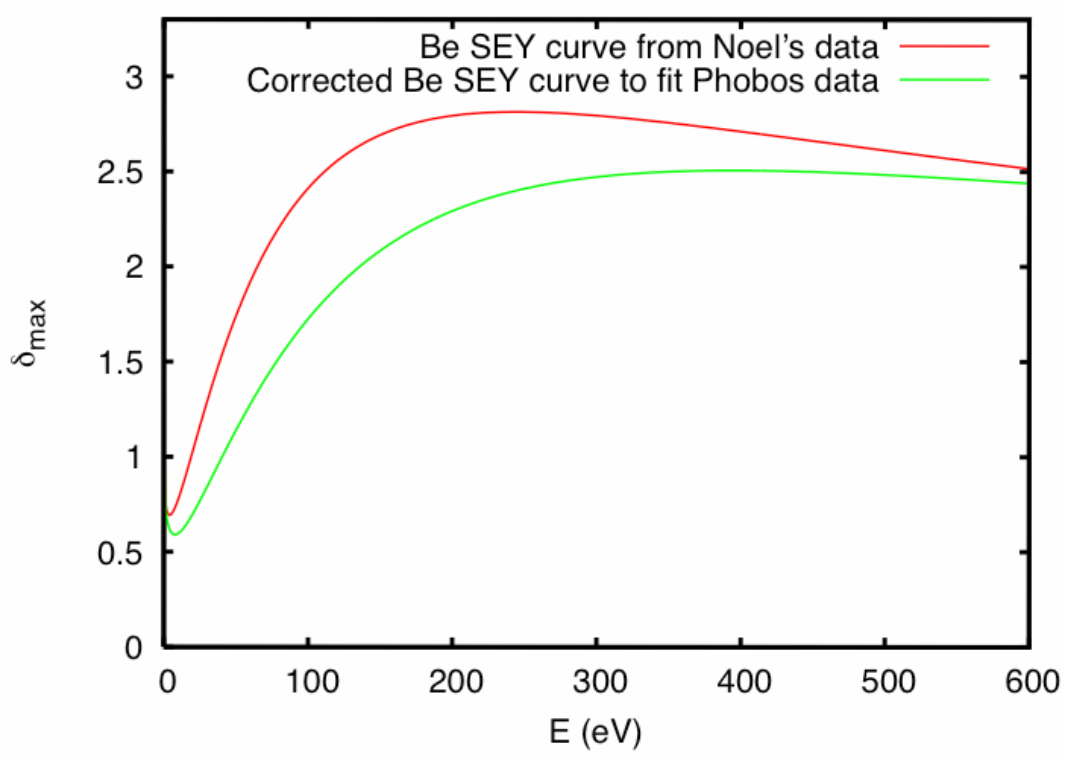

Fig. 5 Secondary emission curves for a Be surface as published in the CERN note [Hilleret00] (red) and as needed to explain the background problem in Phobos (green). Note that both curves have been corrected in the low energy range to account for elastic reflection [Cimino04]. 
The energy spectrum of the electrons hitting the pipe wall, exhibits a long tail towards energies higher than $200 \mathrm{eV}$ for short bunches, but drops significantly around $200 \mathrm{eV}$ for long bunches (see next section). Thus, increasing $\mathrm{E}_{\max }$ and lowering slightly $\delta_{\max }$ creates a situation in which trains of short bunches can produce "more" electron cloud (i.e. forming faster and saturating at higher values) than trains of long bunches prior to rebucketing. In the extreme case, compressed bunches can cause an electron cloud to build up, whereas longer bunches cannot.
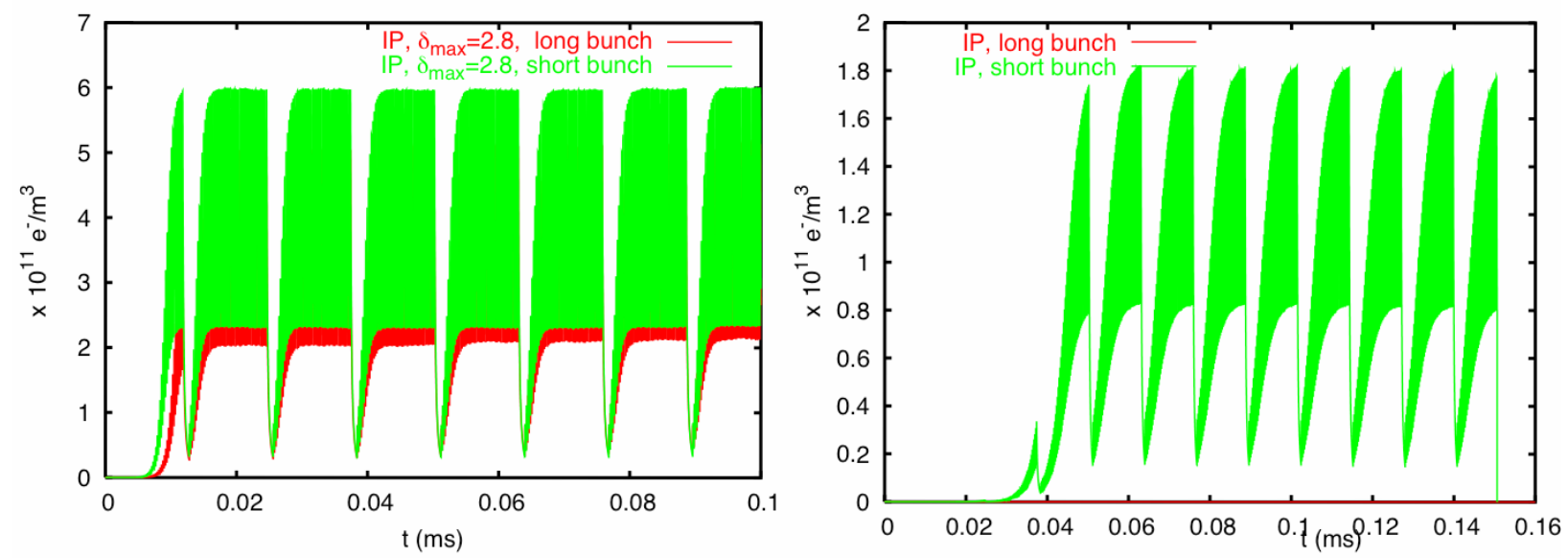

Fig. 6 Electron cloud build up at the IP (6-bucket bunch spacing and double current) before and after rebucketing. SEY parameters are $\left(\mathrm{E}_{\max }=250 \mathrm{eV}\right.$ and $\left.\delta_{\max }=2.8\right)$ for the simulation on the left side, and $\left(\mathrm{E}_{\max }=250 \mathrm{eV}\right.$ and $\left.\delta_{\max }=2.5\right)$ for the simulation on the right side.

At the IP (full bunch spacing and double bunch intensity), lowering $\delta_{\max }$ suppresses the electron cloud production from long bunches (Fig. 6, right side). If in addition also $\mathrm{E}_{\max }$ is shifted to higher values, the electron cloud formation disappears even with short bunches (see Fig. 7, left). For the non-uniform bunch spacing at the end of the Be pipe $(12-52 \mathrm{~m})$, we need to use a combination of SEY parameters $\left(\delta_{\max }=2.5, \mathrm{E}_{\max }=400 \mathrm{eV}\right)$ shown in Fig. 5 (green curve), to obtain an electron cloud build up for short bunches but not for long bunches (Fig. 7, right).
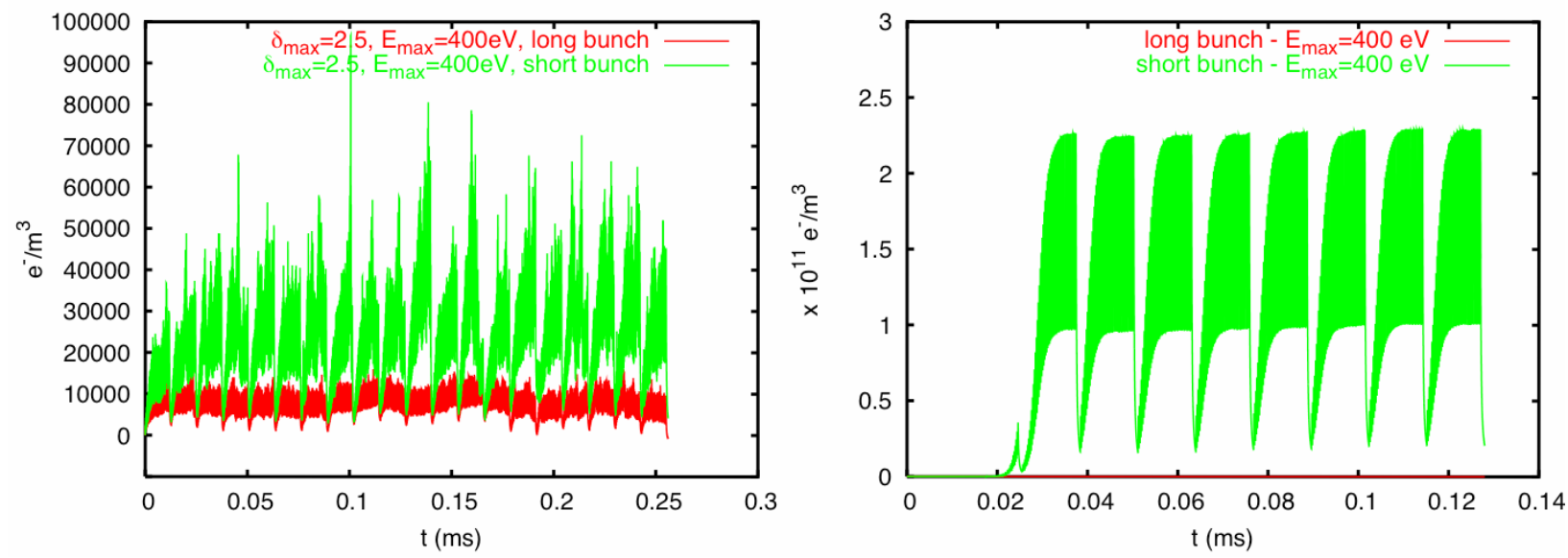

Fig. 7 Electron cloud build up before and after rebucketing with the SEY parameters $\left(\mathrm{E}_{\max }=400 \mathrm{eV}\right.$ and $\delta_{\max }=2.5$ ) at the IP (left side) and at the end of the beryllium pipe (right side).

The study of the bunch pattern with 45 bunches and slightly higher intensity per bunch also shows that no electron cloud is expected to form before rebucketing (long bunches). For short bunches, no electron cloud builds up right at the IP, but at the end of the beryllium pipe, where 
we would be right at the border of the region where an electron cloud could build up (Fig. 8, left). The parameters $\left(\delta_{\max }=2.5, \mathrm{E}_{\max }=400 \mathrm{eV}\right)$ show in fact no electron cloud build up over $0.14 \mathrm{~ms}$ even with the mixed filling pattern $(12,52,12,84)$, which simulates the end of the Be pipe. But if we only lower the $\mathrm{E}_{\max }$ by $50 \mathrm{eV}$, we could still observe electron cloud formation (Fig. 8, left, red curve).

We have then studied the effect of an off-centered beam with the filling pattern for 56 bunches. Long bunches do not induce electron cloud formation, with or without offset. Short bunches at the IP do not cause a cloud build-up. Furthermore, the electron cloud is mitigated for short bunches at the end of the beryllium pipe, too. Figure 8 , right side, shows that offsetting the beam by 8 and $16 \mathrm{~mm}$ is already sufficient to delay the cloud formation and to reduce its saturation value. Offsets of $24 \mathrm{~mm}$ can even suppress the electron cloud build up.
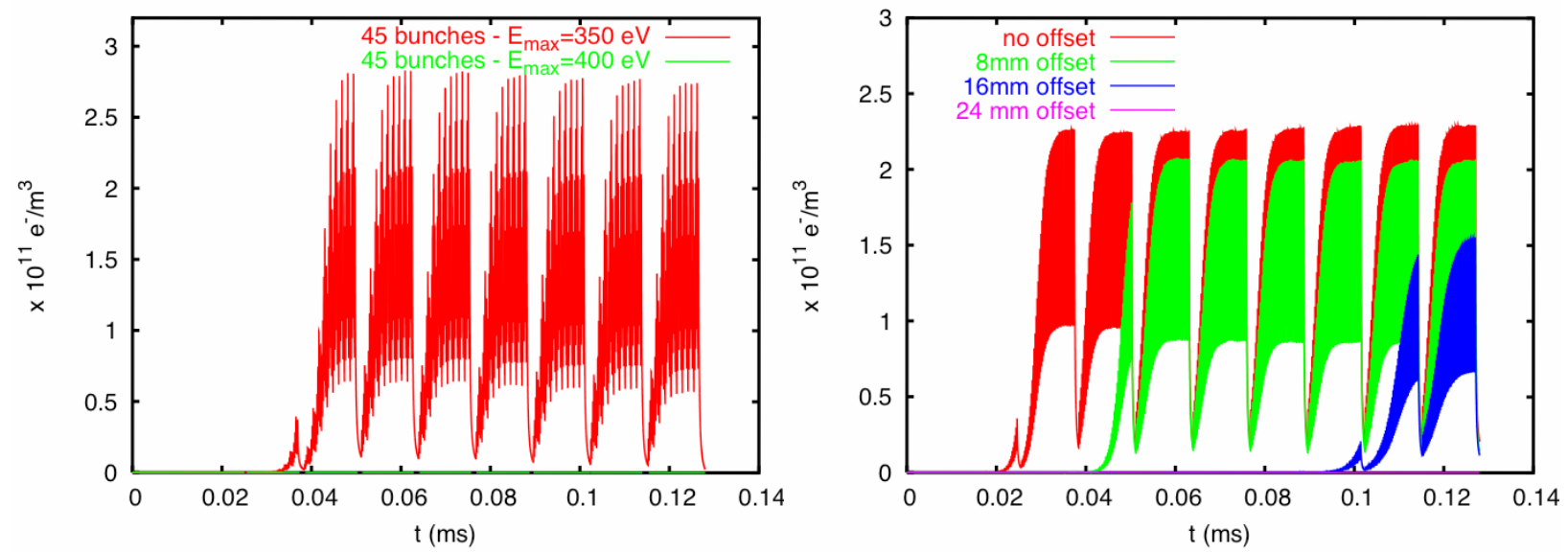

Fig. 8 Electron cloud build up after rebucketing at the end of the beryllium pipe with a 45 bunch filling pattern (left), and with 56 bunches but for different beam offsets (right). The SEY parameters are $\left(\mathrm{E}_{\max }=400 \mathrm{eV}\right.$ and $\left.\delta_{\max }=2.5\right)$, except for the red curve on the left side $\left(\mathrm{E}_{\max }=350 \mathrm{eV}\right)$

Finally, we have made two scans of the cloud formation as a function of the position in the beryllium pipe, and as a function of the bunch intensity. The former tells us which fraction of the beryllium pipe is expected to be affected by electron cloud. The latter provides us with information on the threshold intensity value at which the electron cloud is switched off.

Figure 9, left side, shows that the electron cloud build up becomes somewhat worse at a location $1.5 \mathrm{~m}$ from the pipe end, but then it quickly improves at $3 \mathrm{~m}$ and does not appear at $1.5 \mathrm{~m}$ from the IP. From this simulation we can infer that about a half of the beryllium pipe (about $3 \mathrm{~m}$ starting from both extremities inwards) is affected by a significant electron cloud after rebucketing.

Figure 9, right side, allows us to estimate a threshold current below which the electron cloud does not build up in the PHOBOS beryllium pipe. It is clear that even currents only $8 \%$ smaller than the nominal one can strongly damp the cloud formation and lead to a much lower density of electrons inside the chamber. A current $15 \%$ below the nominal value is sufficient to suppress the electron cloud at any location in the beryllium chamber. 

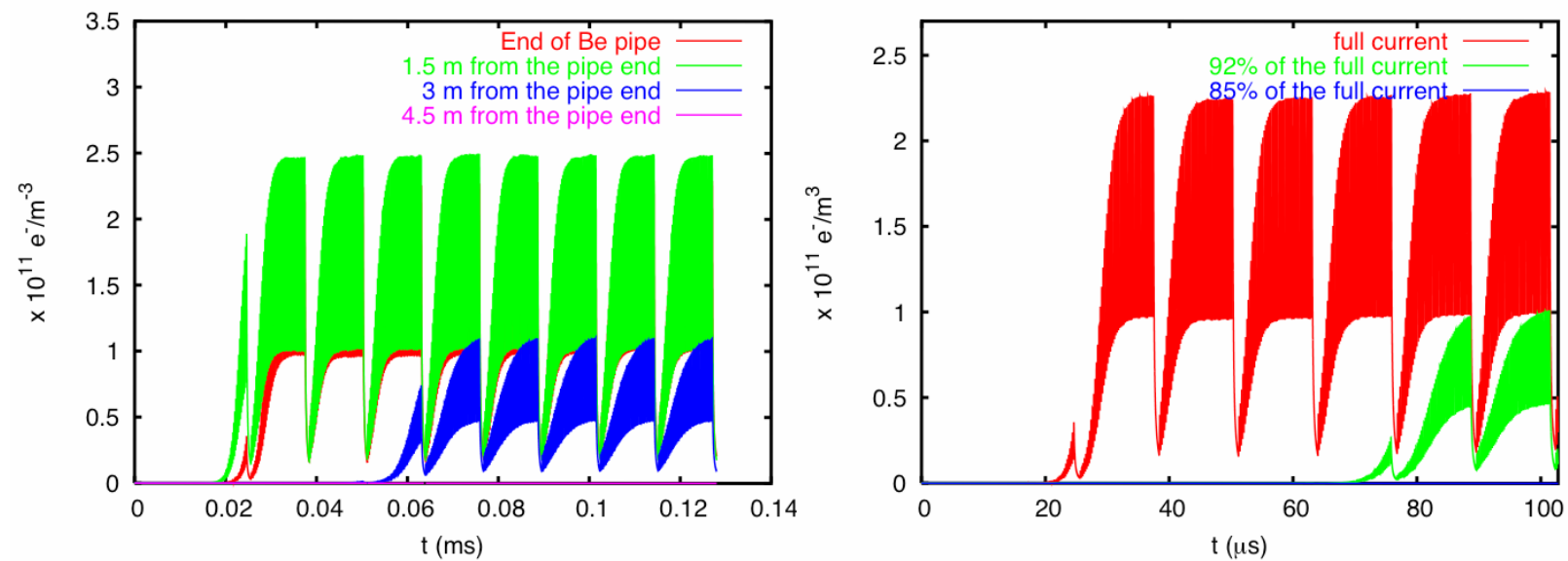

Fig. 9 Electron cloud build up after rebucketing with a 56 bunch filling pattern at different locations in the beryllium pipe (left), and for different bunch intensities (right). In all these simulations, the SEY parameters are $\left(\mathrm{E}_{\max }=400 \mathrm{eV}\right.$ and $\left.\delta_{\max }=2.5\right)$.

\section{Energy spectra and electron fluxes to the wall}

The electron flux into the wall and the energy spectra of the electrons hitting the chamber walls are interesting for two reasons: First, these quantities can be measured with dedicated electron detectors and spectrum analyzers, and can therefore be used for benchmarking simulations against experiments. Second, using the equation for the pressure evolution in steady state [Mustafin03], these values can be used as inputs to estimate the pressure rise. For this purpose, approach and procedure similar to those outlined in Ref. [Rossi02] can be adopted.

Figure 10 shows the energy spectra of electrons hitting the wall for RHIC bunches before and after rebucketing, at the end of the beryllium pipe. The spectra are displayed on two different scales in order to highlight both the dominant low energy component (left side), mainly made of electrons with energies around or below $10 \mathrm{eV}$, and the high energy component of the energy spectrum (right side) extending up to energies of $\sim 370 \mathrm{eV}$.
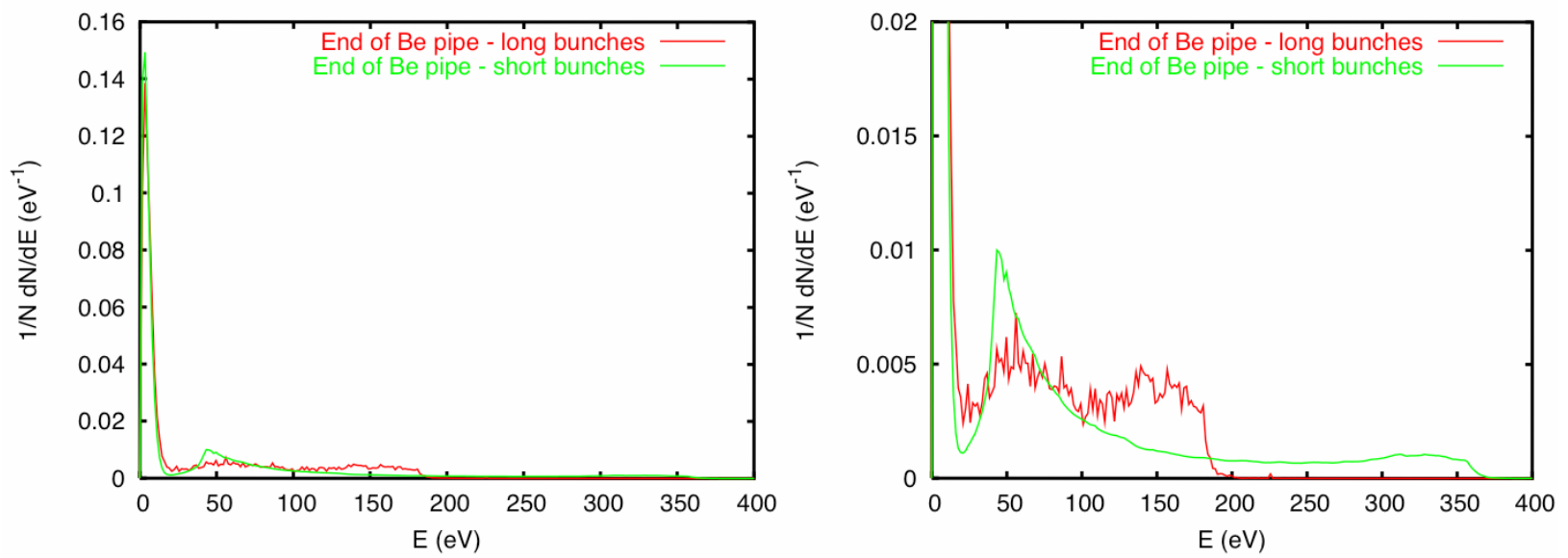

Fig. 10 Energy spectra of electrons hitting the wall, for short and long bunches. The picture on the right shows the high energy part in more detail. 
Figure 11 shows the energy spectra of electrons hitting the beryllium pipe for short bunches and a varying number of ions per bunch. Short bunches can induce multipacting at high currents, while long bunches cannot, because short bunches accelerate electrons to higher energy (close to the $400 \mathrm{eV}$, near the maximum of the SEY). When the bunch intensity is lowered, the accelerating power of the bunch decreases and the electrons will hit the wall with lower energies (see the tails of the energy spectra in Fig. 11).
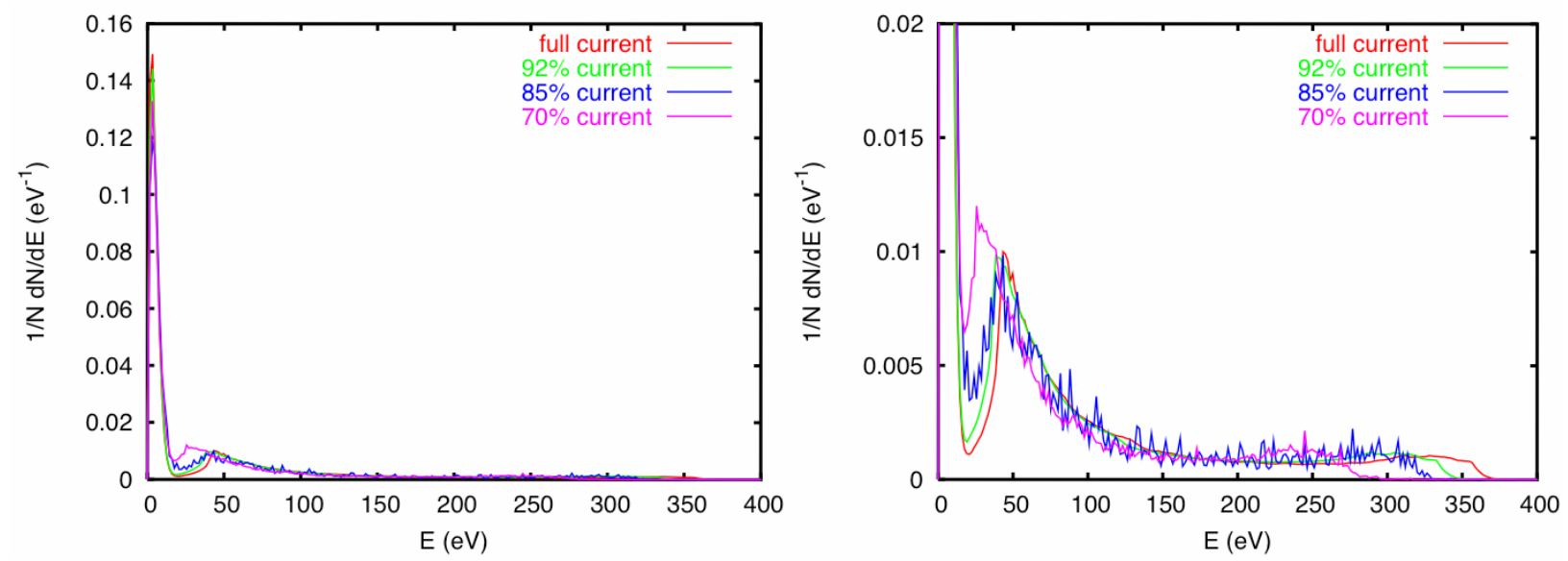

Fig. 11 Energy spectra of electrons hitting the wall for short bunches and different bunch intensities. The picture on the right shows the high energy part in more detail.

More low-energy electrons are also produced with transverse offset beams, and when moving from the end of the beryllium pipe toward the IP (Fig. 12). In the first case, mainly the first peak situated around $50 \mathrm{eV}$ that is smoothed out and moves to the low energy range. In the second case, the effect comes simply from the high-energy tail that is less and less populated.
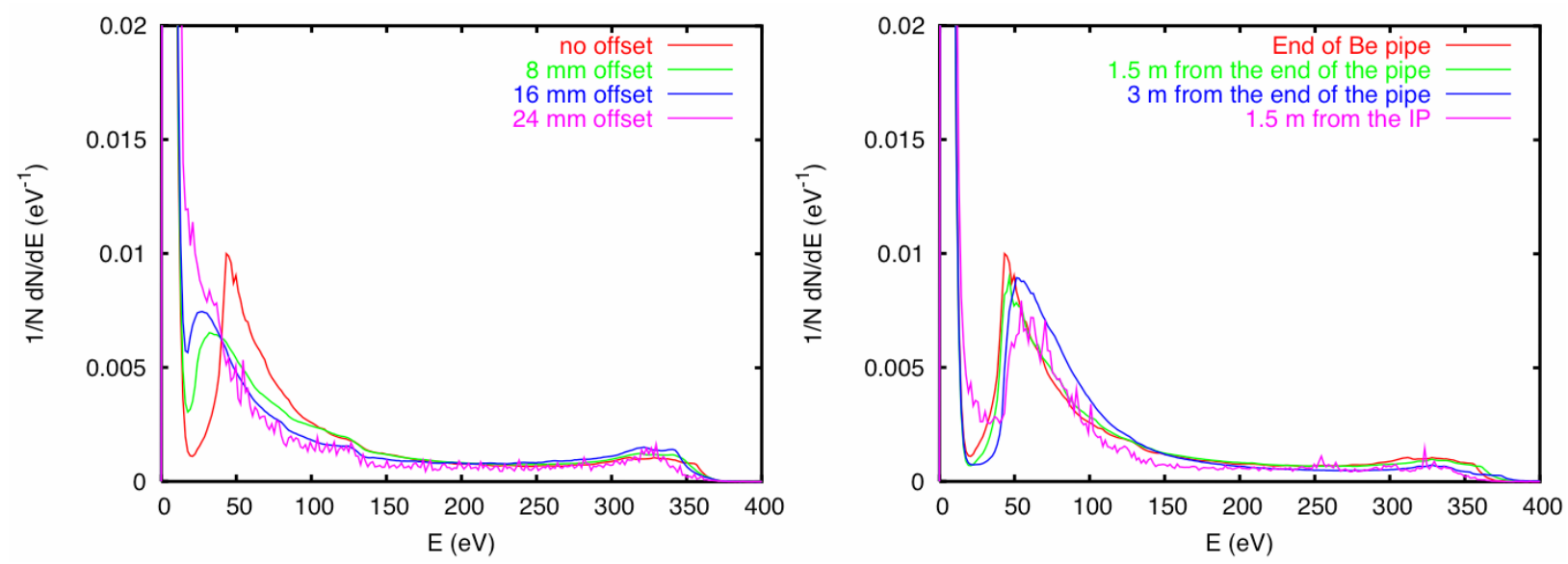

Fig. 12 Energy spectra of electrons hitting the wall for short bunches at the end of the beryllium pipe and different beam offsets (left), and zero offset and different locations in the beryllium chamber (right).

The electron flux into the wall as a function of the position in the beryllium pipe is shown in Fig. 13. An electron cloud is only expected to build up in half of the whole pipe, about $3 \mathrm{~m}$ from either end. Fluxes at the end of the beryllium beam pipe are shown in Fig. 14 as a function of bunch intensity and transverse offset. 


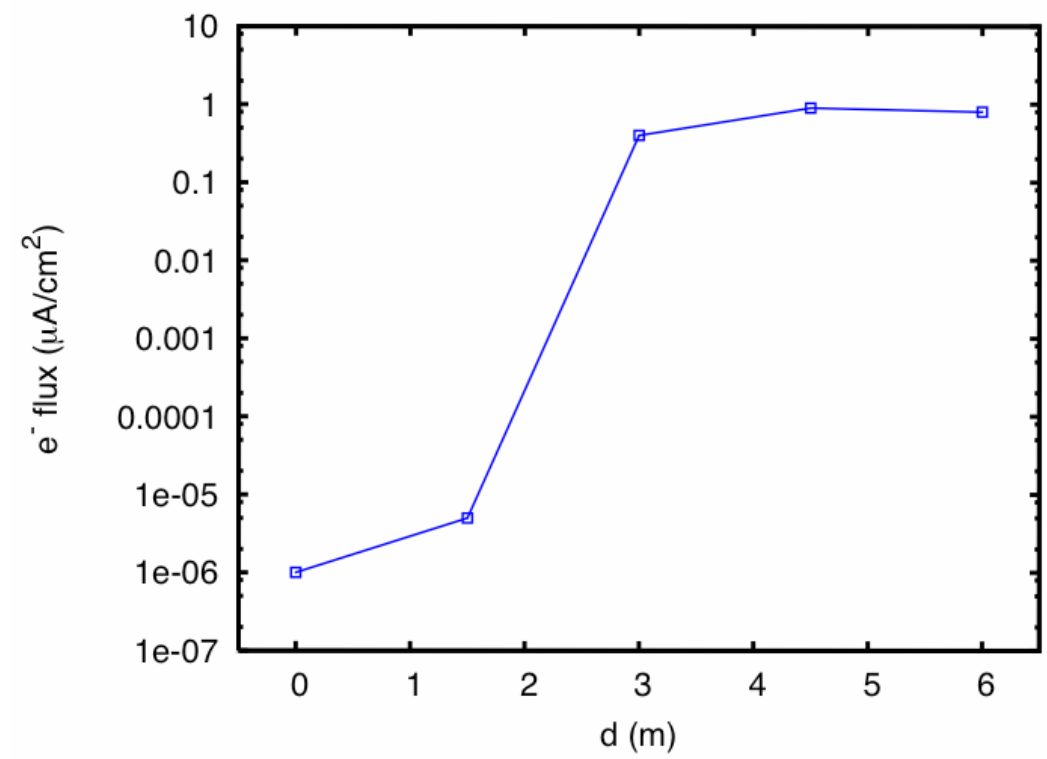

Fig. 13 Electron flux into the beryllium chamber wall as a function of the distance from the IP, for short bunches.
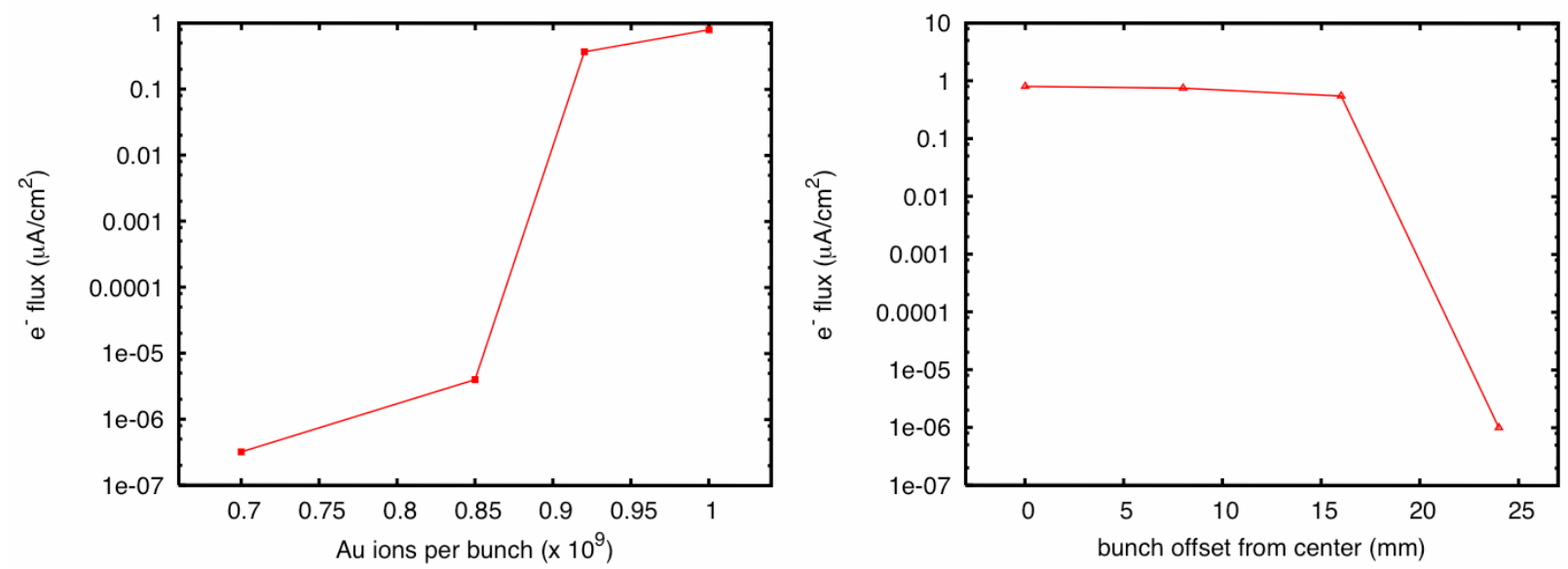

Fig. 14 Electron flux into the wall at the end of the beryllium pipe and for short bunches as a function of the bunch intensity (left) and the transverse beam offset (right).

\section{Discussion and conclusions}

Experimental evidence suggests that an electron cloud causes the background problem at PHOBOS. This is in agreement with simulations, if we assume that the SEY parameters of beryllium are slightly different from those reported [Hilleret00]. Such a deviation is possible because the SEY parameters depend on the history of surface preparation. For example, scrubbing can lower $\delta_{\max }$ and possibly increasing $E_{\max }$ [Cimino04].

Electrons multiply in the beam pipe after rebucketing. The multiplication stops after some time during which the beam intensity and the pressure decrease. The beam intensities at the time of the pressure drop have a large spread around $90 \%$ of the initial intensities. This suggests that other parameters are important. For example, the pressure itself could be part of the feedback 
mechanism that sustains the electron cloud. It is, however, inexplicable that during Run-4 there were a few stores with rather low intensities $\left(\sim 0.5 \times 10^{9} \mathrm{Au}^{79+}\right.$ per bunch), which still exhibited a pressure rise in PHOBOS.

In Run-3, some stores with 110 bunches per ring showed a pressure drop after rebucketing [Zhang04]. Simulations show indeed that with 3 buckets spacing, short bunches cause less dense electron clouds than long bunches. Yet in both cases a dense electron cloud is predicted to build up at any location inside the beryllium chamber with the parameters set that we have used to explain this year's observations.

An important outcome of the simulations is that the electron cloud is most likely to be localized near the ends of the beryllium pipe. This is in agreement with loss monitor signals that are lower at the IP than near the end of the beryllium pipe [Zhang04]. The sections affected by an electron cloud can have a rest gas ionization rate up to about 100 times higher than the beam ionization rate, depending on the electron cloud density [Rumolo01].

Assuming then that electron cloud is responsible for the PHOBOS background problem, possible cures to it are:

- NEG coating of the beryllium pipe. This has the effect of lowering the maximum SEY and therefore suppress any possible cloud formation in the IR. NEG coating on beryllium is currently being tested at CERN and first results are encouraging. The coated test chambers have a low SEY after baking at $180^{\circ} \mathrm{C}$ [Chiggiato04]. This solution requires dismantling and reassembling of the PHOBOS detector. With the electron cloud concentrated near the ends of the beryllium pipe, only the two outer pipes could be coated.

- Solenoids. The full beryllium chamber cannot be wrapped with solenoid coils because of the reduced transparency for collision products. However, the outermost part of the beryllium chamber may be wrapped with solenoids.

\section{Acknowledgements}

The authors would like to thank S.Y. Zhang, M. Blaskiewicz, F. Zimmermann, P. Chiggiato, H.C. Hseuh, U. Iriso, R. Tomás, R. Calaga for stimulating discussions and useful exchange of information. G. Rumolo would also like to express his gratefulness to the whole C-AD Accelerator Physics Group at BNL for the support, for the interesting work and for the friendly atmosphere offered throughout his stay.

\section{References}

[Hilleret00]

[Rumolo02]

[Zhang03]
B. Henrist and N. Hilleret, LHC-VAC Technical Note 00-10 (2000)

G. Rumolo and F. Zimmermann, CERN-SL-Note-2002-016-AP (2002)

S.Y. Zhang, "Experiment background in RHIC deuteron-gold run", BNL C-A/AP/107 (2003) 
[Fischer03] W. Fischer and U. Iriso-Ariz, "Bunch patterns and pressure rise in RHIC", BNL C-A/AP/118 (2003)

[Cimino04] R. Cimino, et al. "Can low energy electrons affect high energy physics accelerators?", GSI-Acc-Note-2004-02-001, SLAC-PUB-10350, LBNL54594, CERN-AB-2004-012 (ABP), presently submitted to PRL.

[Mustafin03] E.Mustafin, et al., NIM A 510 (2003) pp. 199-205.

[Rossi02]

A. Rossi, G. Rumolo and F. Zimmermann, in Proceedings of ECLOUD'02 Workshop, Geneva, edited by G. Rumolo and F. Zimmermann, p. 123

[Zhang04]

S.Y. Zhang, private communication.

[Rumolo01]

G. Rumolo and F. Zimmermann, CERN-SL-Note-2001-014-AP (2001)

[Chiggiato04]

P. Chiggiato, private communication. 